\title{
Flow Simulation in a combined Region
}

\author{
Umurdin Dalabaev* \\ University of World Economy and Diplomacy, Tashkent, Uzbekistan
}

\begin{abstract}
The article deals with the flow in a complex area. The composition of this region consists of a porous medium through the pores of which the liquid moves and a zone without a porous framework (free zone). The flow is modeled using an interpenetrating heterogeneous model. In the one-dimensional case, an analytical solution is obtained. This solution is compared with the solution learned by the move node method. An analysis is made with experimental data with a Brinkman layer. A numerical solution of a two-dimensional problem is also obtained.
\end{abstract}

\section{Introduction}

The flow of a liquid or gas in various media (porous and non-porous) occurs in a variety of applications. Such flows include, for example, the flow of gas and oil in and over a porous medium, underground hydrology, blood flow with stenosis, processes associated with movement through a rough surface, etc. e. Modeling the flow of such problems can be divided into two directions.

The first direction is associated with Darcy's law for a porous layer and the Navier-Stokes equation in the pure region. In [1-6], the flow in the combined region was investigated using the Darcy and Stokes/Navier-Stokes model. In the Darcy model, it is believed that the flow inertia is small, and in the case of highly porous media and in large Reynolds numbers, the results of this model give large errors. At the interface between the porous medium and the isotropic region, the boundary conditions are set [7-10]. In the second approach, which is called one regional, the flow is described by a single equation (equations of the Darcy-Brinkman type) [11-16].

In [17], the flow in the combined region is investigated by a numerical method and with the help of Darcy Brinkman, and a voluminous review of this topic is given.

An experimental study is devoted to [18-19], in which the thickness of the interboundary layer is analyzed.

Here it is proposed to describe the flow inside and outside the porous medium with a single equation using the model of two-phase media

\section{Methods}

Let us consider an interpenetrating model describing the flows of two-phase media [18-19], where the velocity of the discrete phase is neglected. Then the flow of the liquid phase is described by a system of equations (two-dimensional case):

\footnotetext{
*Corresponding author: udalabaev@mail.ru
} 


$$
\begin{aligned}
& \rho \frac{\partial u_{j}}{\partial t}+\sum_{k=1}^{2} \rho u_{k} \frac{\partial u_{j}}{\partial x_{k}}=-f \frac{\partial p}{\partial x_{j}}+\mu \sum_{k=1}^{2}\left(\frac{1}{3} \delta_{j}^{k}+1\right) \frac{\partial}{\partial x_{k}}\left(f \frac{\partial u_{j}}{\partial x_{k}}\right)+ \\
&+\mu \sum_{k=1}^{2}\left(1-\frac{5}{3} \delta_{j}^{k}\right) \frac{\partial}{\partial x_{k}}\left(f \frac{\partial u_{3-j}}{\partial x_{3-k}}\right)-K u_{j}+\rho g_{j} \\
& \sum_{k=1}^{2} \frac{\partial f u_{k}}{\partial x_{k}}=0
\end{aligned}
$$

Here, $u_{j}$ is the $\mathrm{j}$-th component of the flow velocity, $p$ is fluid pressure, $f$ is volume concentration, $\delta_{j}^{k}$ is the Kronecker symbol, $\mu$ is fluid viscosity, $K$ is interaction coefficient, $\rho$ is fluid density, $g_{j}$ is force terms accounting for gravity.

Suppose the equations are transformed into the integral form of representation, then on their basis. In that case, it is possible to study flows both inside and outside porous media, since when $f=1$ we obtain the Navier - Stokes equations for an incompressible fluid. Moreover, these equations are suitable for the entire area under consideration.

\section{Results and Discussion}

Let us consider some one-dimensional problems of applying the model (1), (2).

\subsection{Exact solution}

Let a liquid flow in a flat pipe partially filled with a porous medium. The lower part of the horizontal pipe is filled with a porous medium with height $h$ (pipe height $H$ ). Assuming the flow to be onedimensional and stationary, we obtain from (1), (2)

$$
\mu \frac{d u}{d y}\left(f \frac{d u}{d y}\right)-K u=f \frac{d p}{d x}
$$

In (3), for the parameter K, we use the Carman- Kozeny relation as adopted in porous media:

$$
K=\frac{\mu \cdot f^{2}}{k}
$$

Where $k=\frac{d^{2} f^{3}}{180(1-f)^{2}}$, permeability, $\mathrm{d}$ is the characteristic size of the porous medium.

Let us pass to dimensionless variables by setting $u=\bar{u} U, y=\bar{y} H, x=\bar{x} H, p=\frac{r U^{2}}{\operatorname{Re}} \bar{p}$.

Then equation (3) in dimensionless form at $f=$ const, has the form: 


$$
\frac{d^{2} \bar{u}}{d \bar{y}^{2}}-A \bar{u}=\frac{d \bar{p}}{d \bar{x}}
$$

Here, $A=180(H / d)^{2}(1-f)^{2} / f^{2}$.

In the free zone, the one-dimensional flow satisfies the equation

$$
\frac{d^{2} \bar{u}}{d \bar{y}^{2}}=\frac{d \bar{p}}{d \bar{x}}
$$

Further, in equations (5-6), drop the dash above the variables.

Equation (5) is considered when $0<y<h_{0}$, and equation (6) in $h_{0}<y<1$. Equations are solved under the following boundary conditions. The no-slip conditions for equation (5) in the lower wall and for equation (6) in the upper wall:

$$
u(0)=0, u(1)=0 .
$$

In the interfacial condition, we set the conditions for flow continuity and equality of the shear stress:

$$
u\left(h_{0}-0\right)=u\left(h_{0}+0\right), \frac{d u\left(h_{0}-0\right)}{d y}=\frac{d u\left(h_{0}+0\right)}{d y}
$$

It is easy to obtain an analytical solution to (5) and (6) with the above boundary conditions. Figure 1 shows the analytical solution. The dimensionless pressure drop is taken so that it corresponds to a flow without a porous layer, a unit flow rate. The dotted line corresponds to the solution obtained with a porosity of 0.3 ; the dotted line is 0.5 .

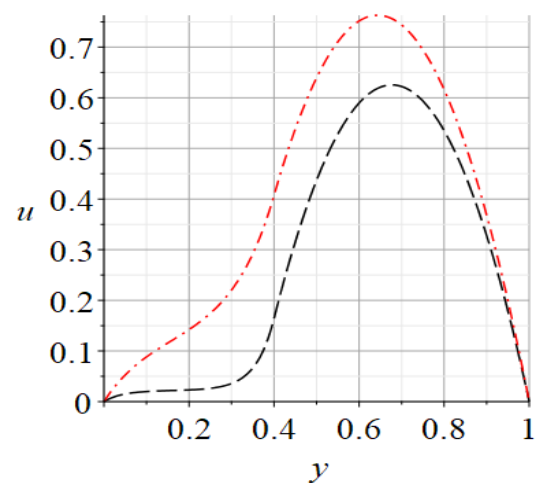

Fig.1. Exact solution: velocity distributions at different porosity values

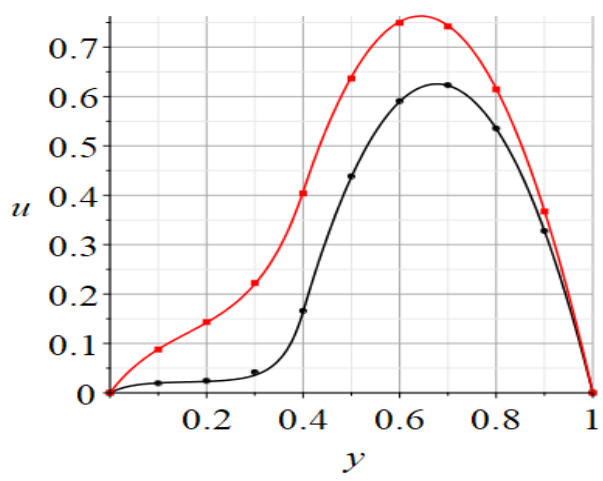

Fig.2. Comparison of the results of accurate and numerical results at different values of porosity

\subsection{Numerical solution}

Consider Eq. (5) for the entire region, and put

$$
f=\left\{\begin{array}{l}
\varepsilon \text { at } 0<y<h_{0} \\
1 \text { at } h_{0} \leq y<1
\end{array}\right.
$$


In this case, equation (5) in the pure region takes the form (6). Thus, equation (5) can be used in the entire region, with porosity (9), while the boundary conditions are fulfilled automatically (in the porous layer, the porosity is taken equal to $\varepsilon$ ). For this purpose, a finite-difference approximation of equation (5) was made, and the calculation was performed using the sweep method (using a special linear system solver designed to take advantage of the tridiagonal structure of the coefficient matrix) in the combined domain.

Figure 2 shows the results of numerical calculations (solid curves, analytical solution, point data, numerical results). This shows that it is possible to make an end-to-end calculation without highlighting the borderline condition.

\subsection{Influence of the pipe roughness thickness on the pressure drop}

To influence the pipe roughness thickness on the pressure drop, we use a solution using an analytical solution. To ensure a unit flow rate of liquid with a unit height of the flow based on Poiselle's law for smooth pipes, it turns out that. Figure 3 shows graphs of the pressure drop depending on the thickness of the porous layer.

Based on figure 3, it can be concluded that the nature of the change in the pressure drop relative to the layer thickness does not depend on the porosity of the layer: it behaves like an "S" shaped function. However, the range of variation strongly depends on the porosity.

\subsection{An approximate analytical solution using a movable node.}

Using the method of movable node [21], one can find an approximate analytical solution to the problem.

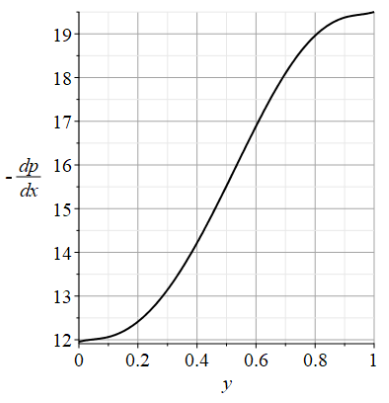

a) $f=0.8$

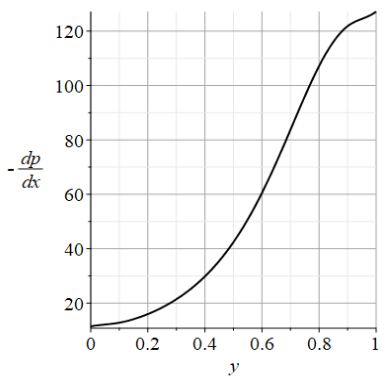

b) $f=0.5$

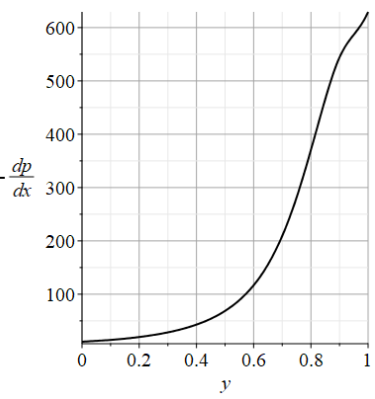

v) $f=0.3$

Fig.3. Pressure drop versus layer thickness

Equation (5.6) is approximated by difference relations:

$$
\begin{aligned}
& \frac{2}{h_{0}}\left(\frac{u_{G}-u}{h_{0}-y}-\frac{u}{y}\right)-A u=\frac{d p}{d x} \\
& \frac{2}{1-h_{0}}\left(\frac{-u}{1-y}-\frac{u-u_{G}}{y-h_{0}}\right)=\frac{d p}{d x}
\end{aligned}
$$


In the difference equations $(9,10)$, the no-slip boundary conditions are used. In equations $(9,10) u_{G}$ is the value of the unknown function on the interfacial boundary. To find $u_{G}$ we use the second interfacial condition (8). Put in (9) $y \rightarrow h_{0}-0$, then we have

$$
\frac{2}{h_{0}}\left(\left.\frac{d u}{d y}\right|_{h_{0}-0}-\frac{u}{h_{0}}\right)-A u_{G}=\frac{d p}{d x}
$$

If in (10) $y \rightarrow h_{0}-0$ then we have:

$$
\frac{2}{1-h_{0}}\left(\frac{-u_{G}}{1-h_{0}}-\left.\frac{d u}{d y}\right|_{h_{0}+0}\right)=\frac{d p}{d x}
$$

Using (8), we obtain

$$
u_{G}=-\frac{h_{0}\left(1-h_{0}\right)}{2+h_{0}^{2}\left(1-h_{0}\right)} \frac{d p}{d x}
$$

Using (13) from (9) and (10), we determine the distribution of velocities in the porous

$$
u=-\frac{y}{2+A y\left(h_{0}-y\right)}\left(h_{0}-y+\frac{2\left(1-h_{0}\right)}{2+h_{0}^{2}\left(1-h_{0}\right)}\right) \frac{d p}{d x} .
$$

and free zones

$$
u=-\frac{1-y}{1-h_{0}}\left(\frac{\left(1-h_{0}\right)\left(y-h_{0}\right)}{2}+\frac{h_{0}\left(1-h_{0}\right)}{2+A h_{0}^{2}\left(1-h_{0}\right)}\right) \frac{d p}{d x}
$$

In fig. 4, the exact and approximate solutions are compared (the solid line is the exact solution, and the dotted line is approximate, obtained using (14) and (15) at $A=40000, h_{0}=0.2$ ).

\begin{tabular}{|r|r|r|r|}
\hline 0.9 \\
0.8 \\
0.7 \\
0.6 \\
$u$ & 0.5 \\
0.4 \\
0.3 \\
0.2 \\
0.1 \\
0
\end{tabular}




\subsection{Comparison with experiment}

In [22], the thickness of the Brinkman boundary layer was experimentally investigated. In this layer, which is located between the Darcy layer and the layer with a pure region, based on experimental data, an empirical formula is obtained

$$
\bar{u}=\frac{u-u_{D}}{u_{\text {int }}-u_{D}}=\exp (\gamma(\bar{y}-1))
$$

Here is $u_{D}$ the Darcy velocity, $u_{\text {int }}$ is the interfacial velocity, $\gamma=3-4(\bar{y}-1)$, Figure 5 shows a comparison of the results obtained based on (5) and (6) (solid line) and (16) for the transition layer.

To obtain a visual representation of the solution to the problem in the combined domain, a numerical calculation was performed using equations (1-2) based on a similar SIMPLE algorithm [19].

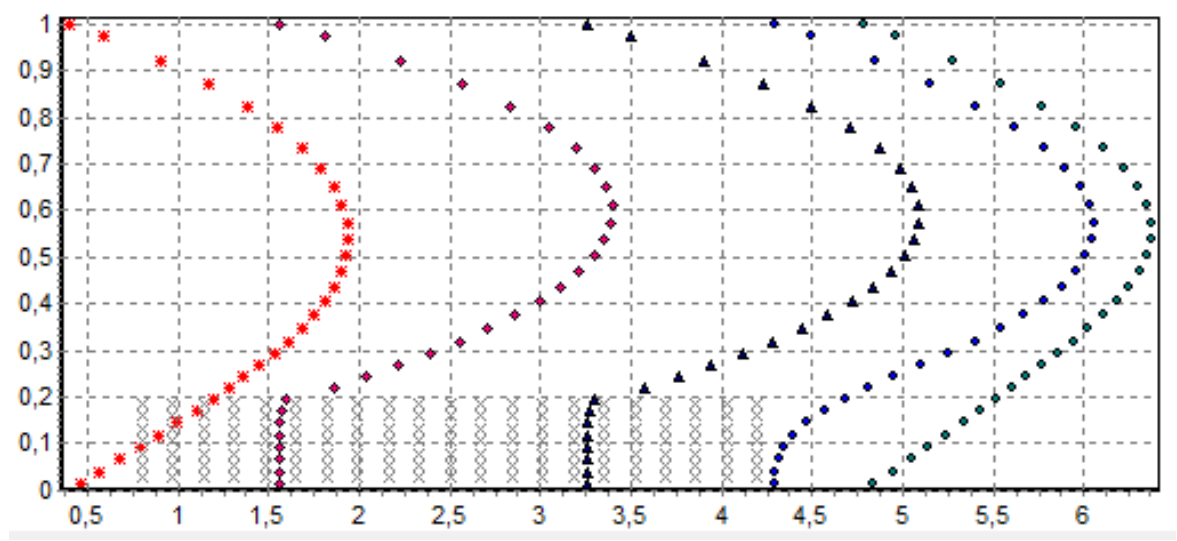

Fig.6. Longitudinal velocity profiles at different sections $(f=0.4)$.

In figure 6 , the porous layer is located in the region $0.8 \leq x \leq 4.2 ; 0 \leq y \leq 0.1$ porosity $f=0.4$. At the entrance, a parabolic law is given; in the sections inside the porous layer, the profile is flattened, and a rapid increase in velocity begins to occur $(\operatorname{Re}=21)$. 


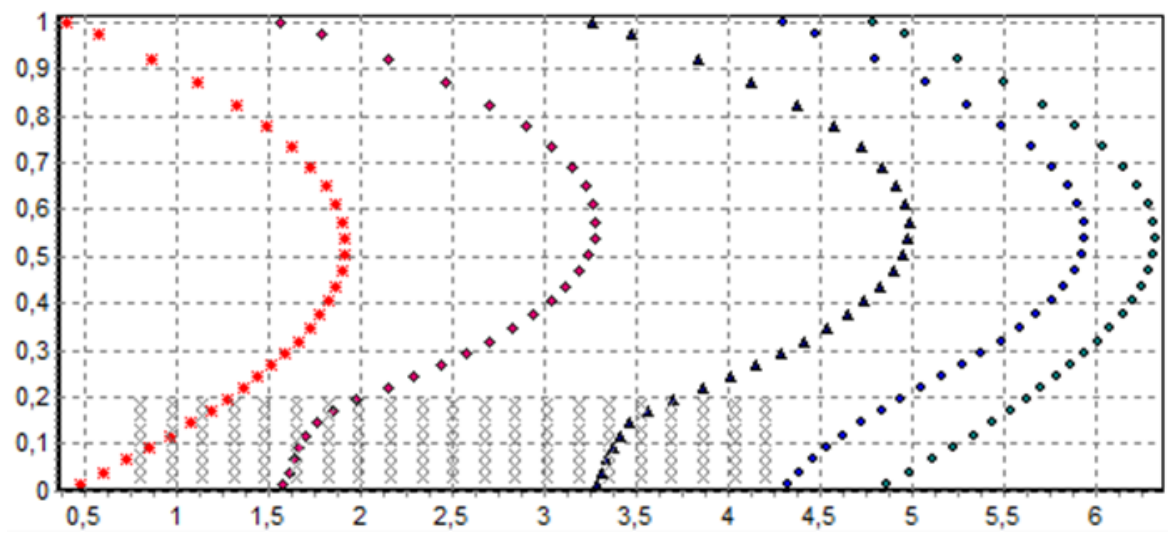

Fig.7. Longitudinal velocity profiles at different sections $(f=0.8)$.

In figure 7 numerical results, the porosity of the layer is 0.8 . In this case, Darcy's law does not hold inside the layer. The irregularity of the velocity profile covers the entire porous region. Visual comparison with experiment [23] gives good results concerning the interfacial layer.

\section{Conclusions}

Rakhmatulin's model can be successfully applied to describe the flow in the combined region. An approximate analytical solution obtained on the basis of the method of movable nodes can be used to describe the flow for one-dimensional problems; to obtain more accurate approximate solutions, it is possible to achieve multipoint movable nodes. The results of the study are in good agreement with the experimental results. The results of test calculations and the influence of grid parameters, reflecting the validity of the application of the model and the proposed numerical approach, are presented.

\section{References}

1. William Jj. Llayton, Friedhelm Schieweck, and Ivan Yotov, Coupling fluid flow with porous media flow, SIAM J. NUMER. ANAL., 40(6), pp. 2195-2218

2. Dayle Jogie1, Balswaroop Bhatt, Flow of immiscible fluids in a naturally permeable channel, International Journal of Pure and Applied Mathematics, 78(3),pp 435-449 (2012)

3. Kirill Tsiberkin, Ekaterina Kolchanov, and Tatyana Lyubimov, Verification of the boundary condition at the porous medium-fluid interface, EPI Web Conferences 114, 02125 (2016) DOI: 10.105/epjconf/ 201611402125. (2016).

4. Philippe Angot, Well-posed stokes, brinkman and stokes/darcy coupling revisited with new jump interface conditions, Mathematical Modelling and Numerical Analysis, ESAIM: M2AN 52 pp 1875-1911, https://doi.org/10.1051/m2an/2017060. (2018)

5. Matthias Ehrhardt, An Introduction to Fluid-Porous Interface Coupling, Progress in computational physics, 2:3-12, 2000..

6. Fernando A. Moralesa,, Ralph E.Showalterb, A Darcy-Brinkman model of fractures in porous media, Journal of Mathematical Analysis and Applications, J. Math. Anal. Appl. 452 pp 1332-1358. (2017).

7. Beavers G. S., Joseph D. D., Boundary conditions at a naturally permeable wall, J. Fluid Mech., 30 pp. 197-207. (1967), 
8. Saffman P. G. On the Boundary Condition at the Surface of a Porous Medium. Stadies in Applied Mathematics, pp.93-101, https://doi.org/10.1002/sapm197150293. (1971)

9. J. Alberto Ochoa-Tapia, J. Whitaker.S. Momentum transfer at the boundary between a porous medium and a homogeneous fluid-I. Theoretical development, International Journal of Heat and Mass Transfer, 38(14), September 1995, pp 2635-2646, https://doi.org/10.1016/0017-9310(94)00346-W. (1995)

10. J. Ochoa-Tapia, J.A., Whitaker, S., Momentum jump condition at the boundary between a porous medium and a homogeneous fluid: inertial effect. J. Porous Media 1, pp 201-217. (1998).

11. Yu, P., T.S., Zeng, Y. Low, Y. T. , A Numerical for flows in porous and homogenous fluid domains coupled at the interface by stresss jump. Int. J. Numer. Meth. Fluids 53, pp 1755-1775, https://doi.org/10.1002/fld.1383

12. Oleg Iliev and Vsevolod Laptev. On numerical simulation of flow through oil filters. Computing and Visualization in Science, 6(2) pp 139-146, Mar 2004. ISSN 14330369. doi: 10.1007/s00791-003-0118-8. URL https://doi.org/10.1007/s00791-0030118-8. (2004).

13. Ershin Sh. A, Zhapbasbayev U. K., Model of Turbulent Motion of Incompressible Liquid in Apparatus with a Permeable Partition, Publishing House of the SB RAN: Applied Mechanics and Technical Physics

14. Gaev E. A. Shikhaliev S. Z, Numerical study of fluid inlet into a channel with a linear easily permeable roughness, Applied hydrodynamics, 4, (76), pp. 32-39.( 2002).

15. Dalabaev, U, Numerical investigation of the character of the lift on a cylindrical particle in Poiseuille flow of a plane channel, 6 November 2011, https://doi.org/10.1007/s10891-011-0609-2. (2011).

16. Dalabaev, U. Structure of flow through an immovable granular layer. J Eng Phys Thermophys 70, pp 379-382 https://doi.org/10.1007/BF02662134. (1997).

17. Kazunori Fujisawa, Akira Murakami, Numerical analysis of coupled flows in porous and fluid domains by the Darcy-Brinkman equations, Soils and Foundations, 58, (5), October 2018, pp 1240-1259 https://doi.org/10.1016/j.sandf.2018.07.003. (2018).

18. Faizullaev, D. F, Laminar Motion of Multiphase Media in Conduits, Springer US, ISBN 978-1-4899-4832-8 (1969).

19. Nigmatulin, R. I., Fundamentals of the mechanics of heterogeneous media, Moscow, Izdatel'stvo Nauka, p 336. In Russian. (1978).

20. Patankar S. Numerical Heat Transfer and fluid Flow, ISBN 9780891165224 Published January 1, by CRC Press.( 1980).

21. Dalabaev U. Computing Technology of a Method of Control Volume for obtaining of the Approximate Analytical Solution one-dimensional Convection-diffusion Problems. Open Access Library Journal, 5: $1104962 .$. https://doi.org/10.4236/oalib.1104962 (2018).

22. Mohammad Reza Murad, Arzhang Khalili, Transition layer thickness in a fluid-porous medium of multi-sized spherical beds, Exp. Fluids 46:323-330, DOI 10/1007/s00348008-0562-9. (2009)

23. Afshin Goharzadeh, Arzhang Khalili, and Bo Barker Jorgensen, Transition layer thickness at a fluid-porous interface, Physics of fluids 17: 057102, DOI 10.1063/1.1894756. (2005) 